\title{
Astrophysical tau neutrinos and their detection by large neutrino telescopes
}

\author{
Edgar Bugaev, ${ }^{1}$ Teresa Montaruli, ${ }^{2,3}$ and Igor Sokalski ${ }^{3,1}$ \\ ${ }^{1}$ INR, 60th October Anniversary Prospect 7a, 117312, Moscow, Russia \\ 2 Physics Department, Bari University, Via Amendola 173, 70126 Bari, Italy \\ ${ }^{3}$ INFN/Bari, Via Amendola 173, 70126 Bari, Italy
}

(November 1, 2018)

\begin{abstract}
We present results of the detailed Monte Carlo calculation of the rates of double-bang events in $1 \mathrm{~km}^{3}$ underwater neutrino telescope with taking into account the effects of $\tau$-neutrino propagation through the Earth. As an input, the moderately optimistic theoretical predictions for diffuse neutrino spectra of AGN jets are used.
\end{abstract}

\section{INTRODUCTION}

During these years there have been many discussions $[1,2]$ on the possibilities of very high energy $\tau$-neutrino detection by large neutrino telescopes, which would prove oscillations of muon neutrinos of astrophysical origin ("astrophysical long baseline experiment" [3]).

It is known now that neutrino oscillations definitely exist. Nevertheless, the detection of astrophysical $\tau$ neutrinos is still very interesting and useful, e.g., for a better understanding of neutrino properties as well as properties of astrophysical sources [4].

Intrinsic flux of $\tau$-neutrinos from a typical source in which there is acceleration of protons and production of neutrinos through $p p$ - and $p \gamma$-reactions is very small. The dominant channel of $\tau$-neutrino birth is through the inclusive production of charmed mesons [5],

$$
p p, p \gamma \longrightarrow D_{S}^{+}+X, \quad D_{S}^{+} \longrightarrow \tau \nu_{\tau}
$$

and the smallness of $\nu_{\tau}$-flux follows from the relations

$$
\frac{\sigma_{\gamma p \rightarrow D X}}{\sigma_{\gamma p \rightarrow \pi X}} \lesssim 10^{-3}, \frac{B r\left(D_{S}^{+} \rightarrow \nu_{\tau}\right)}{B r\left(\pi \rightarrow \nu_{\mu}\right)} \sim 7 \cdot 10^{-2} .
$$

Therefore, the production of $\nu_{\tau}$ at source is negligible, and if all muons can decay, it can be expected for the 3 flavors the following proportion:

$$
\Phi_{s t d}^{0}=\left\{\phi_{e}^{0}, \phi_{\mu}^{0}, \phi_{\tau}^{0}\right\}=\left\{\frac{1}{3}, \frac{2}{3}, 0\right\} .
$$

Assuming maximal atmospheric mixing and $U_{e 3}=0$, that is:

$$
\sin \theta_{13}=0, \quad \sin 2 \theta_{23}=1
$$

one obtains, after averaging over many oscillations, the ideal equipartition between neutrino flavors in the astrophysical neutrino flux at Earth,

$$
\Phi_{\text {std }}=\left\{\frac{1}{3}, \frac{1}{3}, \frac{1}{3}\right\},
$$

independently on the value of the solar mixing angle $\theta_{12}$. Moreover, in the case when there is maximal atmospheric mixing and $U_{e 3}=0$, for any proportions between flavors at the source one has, after propagation, the equality $\phi_{\mu}=\phi_{\tau}[4]$.

The experimental verification of the relation $\phi_{\mu}=\phi_{\tau}$ by detection of astrophysical diffuse fluxes of $\nu_{\tau}$ and $\nu_{\mu}$ with flavor identification capabilities is very important because the inequality $\phi_{\mu} \neq \phi_{\tau}$ is predicted in some exotic scenarios, e.g. [4]: i) if CPT invariance is violated in the neutrino sector and the neutrino and antineutrino mixing matrices do not coincide, $\phi_{\mu}$, in general, is not equal to $\phi_{\tau}$, ii) if neutrinos can decay [6] en route from the sources to the Earth, the neutrino fluxes and flavor ratios are very sensitive to uncertanties in the neutrino mixing matrix and strongly depend on the hierarchy of neutrino masses. So, the inequality $\phi_{\mu} \neq \phi_{\tau}$ would reveal unconventional neutrino physics.

Inside of some astrophysical and cosmological objects there are processes in which very energetic quarks and gluons fragmenting into jets of hadrons are expected to be produced. Even the usual inclusive spectrum of high energy $p p$ - or $p \gamma$-collisions always contains the jet component (described well by perturbative QCD). Jets can appear in decays of (hypothetical) supermassive particles which, in turn, are produced in decays of topological defects [7], in processes of superheavy dark matter annihilation inside of stars [8], in a process of Hawking evaporation of primordial black holes [9]. It was shown in [10] that decays of top quarks contained in the jets lead to production of $\tau$-neutrino flux from the jets, which is of the same order as $\nu_{\mu}, \nu_{e}$ fluxes (at large neutrino energies, close to the value of the jet mass). This means that the intrinsic $\tau$-neutrino flux from the sources in which the jet phenomena are essential is, in general, not suppressed (in comparison with intrinsic $\nu_{\mu}, \nu_{e}$ fluxes).

We studied in this paper the possibility of detection of extragalactic diffuse $\tau$-neutrino fluxes by large $\left(\sim 1 \mathrm{~km}^{3}\right)$ underwater neutrino telescopes. In Sec.II we discuss the general problems connected with the detection of very high energy $\tau$-neutrinos $\left(E_{\nu} \gtrsim 10^{6} \mathrm{GeV}\right)$. In Sec.III we present the diffuse neutrino spectra for the AGN jets chosen for the numerical calculations. Details of the calculations, results and conclusions are given in Sec.IV. 


\section{DETECTION OF $\tau$-NEUTRINOS}

The main feature of charge current interactions of $\tau$ neutrinos is the fact that $\tau$-leptons decay long before they lose a large fraction of their energy. This leads to the absence of absorption of $\tau$-neutrinos during their propagation through the Earth and, as a consequence, to the characteristic modification of the neutrino spectrum after crossing the Earth (there is a pile-up of events with energies around $\sim 100 \mathrm{TeV}$, if the incident neutrino spectrum is not too steep). At neutrino energies $\sim 10^{6}-10^{7} \mathrm{GeV}$ $\tau$-lepton track-length becomes larger than $\sim 100 \mathrm{~m}$ (in water), it becomes possible to identify $\tau$-neutrino interactions in large neutrino telescopes by selection of "double-bang" or "lollipop" events [1]. A double-bang event consists of two showers and a $\tau$-lepton track connecting them. The first (hadronic) shower is initiated by the charge current interaction of $\tau$-neutrino and the second one (hadronic or electromagnetic) is produced by the $\tau$-lepton decay. In lollipop events one of these showers is not detected (e.g., if the $\tau$-neutrino interaction takes place outside of the detector).

The detection of events with double-bang structure would reliably indicate the appearance of $\tau$-neutrinos in the detector because this signature is unique. However, the probability of observing a double-bang event $P_{D B}$ decreases with neutrino energy for $E_{\nu}>10^{8} \mathrm{GeV}$ (due to $\tau$-lepton range increase). The lollipop structure is not so spectacular although the observation probability $P_{L}$ of lollipop events is at $E_{\nu} \sim 10^{8} \mathrm{GeV}$ considerably (by a factor of 10 [11]) larger than $P_{D B}$.

Diffuse astrophysical and cosmological neutrino fluxes in the ultra-high energy region, $E_{\nu}>10^{9} \mathrm{GeV}$, (GZK neutrinos (see, e.g., [12]), hypothetical neutrino fluxes from topological defects [13], from Z-bursts [14] etc.) are too small and inaccessible for a study by underwater neutrino telescopes. Registration of $\tau$-neutrinos of such energies can be accomplished by detecting the air showers caused by $\tau$-leptons produced by neutrino-nucleon interactions far away from the detector ("Earth-skimming idea" [15]). The huge modern air shower detectors (e.g., the HiRes [16] or the Pierre Auger detector [17]) could be suitable for such experiments, but expected event rates are low.

\section{DIFFUSE NEUTRINO SPECTRA FROM AGN JETS}

The concrete calculations were carried out with two theoretical diffuse neutrino spectra from AGNs.

1. The curve denoted by "MPR(limit)" in Fig.1 is the generic upper bound on the diffuse neutrino spectrum from AGN jets obtained by Mannheim, Protheroe, and Rachen [18]. This limit was found using the assumption that the AGN source is not completely optically thin for a cosmic ray flux: due to opacity effects there is a

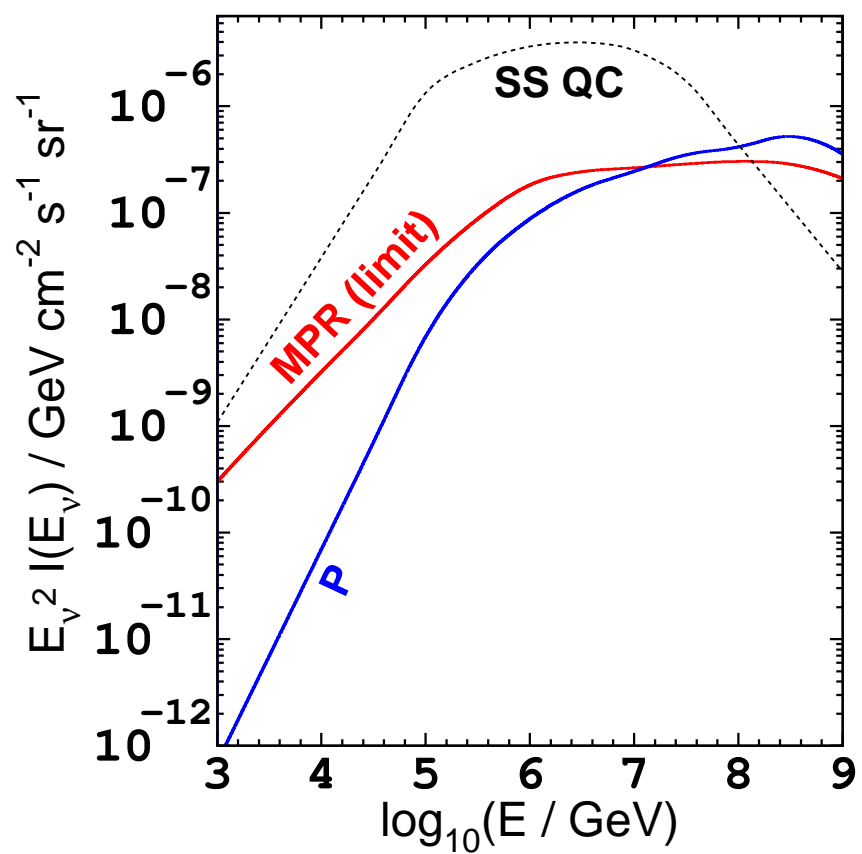

FIG. 1. Different theoretical predictions for diffuse neutrino $\left(\nu_{\mu}+\tilde{\nu}_{\mu}\right)$ spectra from AGNs. MPR(limit): upper bound for $\nu_{\mu}$ flux from AGN jets [18], P: $\nu_{\mu}$ flux for proton blazar model of [21], SSQC: prediction of $\nu_{\mu}$ flux from radio-quiet AGNs [22].

spectral break between $10^{7} \mathrm{GeV}$ and $10^{11} \mathrm{GeV}$ in the escaping cosmic ray spectrum (the resulting cosmic ray spectrum is a combination of several model spectra with different values of the spectral break and with the fixed $E_{\max }\left(E_{\max }=10^{11} \mathrm{GeV}\right)$, and the normalization of the resulting spectrum is such that the cosmic ray intensity does not exceed the proton spectrum estimated from observations). Neutrinos are mostly produced in decays of mesons from accelerated proton interactions with synchrotron photons emitted by high energy electrons in the jets. It is noted in [18] that various models of AGN jets (see, e.g., [19-21]) predict diffuse neutrino spectra which are compatible with the MPR limit (at the important neutrino energy range below $\left.\sim 10^{7} \mathrm{GeV}\right)$.

2. The model by Protheroe [21] (the curve denoted by "P" in Fig.1) is the optically thick proton blazar model, in which it is assumed that the target for the pion production is provided by the thermal UV photons emitted from the accretion disc rather than by the synchrotron photons produced in the jets. The diffuse neutrino spectrum is normalized to the experimental data on $\gamma$-ray background. It is seen from the figure that the prediction of this model is, indeed, not in the contradiction with the MPR bound, if $E_{\nu} \leq 10^{7} \mathrm{GeV}$. The proton blazar model, developed by Mannheim [19] (in which accelerated protons interact with the synchrotron radiation of jet electrons) predicts very similar diffuse neutrino spectrum.

One should note that the prediction given by Stecker and Salamon [22] for diffuse neutrino spectrum from 
radio-quiet $\mathrm{AGNs}$ is much more optimistic (SSQC curve in Fig.1) at neutrino energies $E_{\nu} \sim 10^{6}-10^{7} \mathrm{GeV}$. Their model, however, predicts a non-thermal spectrum of Xrays at energies below $\sim 500 \mathrm{KeV}$ (these X-rays result from reprocessing of gamma-rays from pion decays by electromagnetic cascades due to the high photon density near the black hole) while the observed X-ray spectra of radio-quiet AGNs (as well as the spectrum of the diffuse $\mathrm{X}$-ray background) turn over steeply below $\sim 100 \mathrm{KeV}$ with no sign for a nonthermal component [23]. Therefore, the existing X-ray data cannot be used for a normalization of the radio-quiet AGN neutrino spectrum (authors of [22] normalized their spectrum, rather arbitrarily, on $30 \%$ of the diffuse X-ray background). This is the reason why we did not use SSQC flux in our calculations. Besides, SSQC neutrino flux is now almost excluded by measurements on AMANDA [24] and Baikal [25] neutrino telescopes.

\section{RESULTS AND DISCUSSIONS}

First estimates of double-bang event rates in $\mathrm{km}^{3}$ neutrino telescope were done in [26], for down-going $\tau$ neutrinos only. Detailed calculations of $\tau$-neutrino propagation through the Earth carried out by Dutta et al. [27] (using power law incident neutrino spectra) showed that, in spite of the absence of neutrino absorption, the contribution of lower hemisphere events to the total event rate in a telescope cannot be too large. This is due to a strong decrease of neutrino energies during propagation. It was concluded in [27] that the most promising way to detect $\tau$-neutrino astrophysical fluxes is an analysis of shower events in the telescope.

In this work we present results on Monte Carlo calculations of rates of double-bang events in a $1 \mathrm{~km}^{3}$ telescope for both hemispheres using the diffuse neutrino spectra shown on Fig.1. We simulate both DIS CC and NC neutrino interactions for all flavors in the energy range $10^{3} \mathrm{GeV}<E_{\nu}<10^{9} \mathrm{GeV}$ using the CTEQ3_ DIS structure functions. For $\tau$-lepton propagation we used the MUM code [28] that was updated accounting for the corrections for photonuclear interactions of $\tau$-leptons [29]. Some details of the calculations and the resulting neutrino fluxes of all flavors after propagation through the Earth were presented in our previous work [30].

The rate of totally contained double-bang events is given by the formula

$$
\begin{array}{r}
N=2 \pi \rho N_{A} \int_{-1}^{1} \int_{E_{\min }}^{\infty} V_{e f f}\left(E_{\nu_{\tau}}, \theta\right) I\left(E_{\nu_{\tau}}, \theta\right) \times \\
\sigma^{C C}\left(E_{\nu_{\tau}}\right) d E_{\nu_{\tau}} d(\cos \theta) .
\end{array}
$$

Here, $N_{A}$ is the Avogadro number, $\rho$ is the density of the medium (we use $\rho=1 \mathrm{~g} \mathrm{~cm}^{-3}$ which is close to sea water/ice density), $I\left(E_{\nu_{\tau}}, \theta\right)$ is the differential $\nu_{\tau}$ flux entering the detector at zenith angle $\theta, \sigma^{C C}$ is the total deep inelastic neutrino cross section (charge current part). $V_{\text {eff }}$ is the effective volume given by the relation:

$$
V_{e f f}\left(E_{\nu_{\tau}}, \theta\right)=S_{p}(\theta)\left(L-R_{\tau}\left(E_{\nu_{\tau}}\right)\right),
$$

where $S_{p}$ is the projected area for tracks generated isotropically in azimuth at the fixed $\theta$ directions on a detector of parallelepiped form, $L$ is the geometrical distance between entry and exit point, $R_{\tau}$ is the $\tau$ lepton range. The minimum value of the neutrino energy, $E_{\text {min }}=2 \cdot 10^{6} \mathrm{GeV}$,corresponds to a $\tau$-lepton range of $R_{\tau}^{\min }=70 \mathrm{~m}$.

The double-bang topology can be considered as a background free signature of $\tau$-neutrino appearance [1] since there should be no atmospheric muons which could produce in the detector 2 showers with comparable amounts of photons. Nevertheless, this reasonable assumption needs to be verified through a full simulation.

Concrete calculations were done for two geometries of the neutrino detector:

$$
\begin{aligned}
& 1 \times 1 \times 1 \quad \mathrm{~km}^{3} \quad \text { (IceCube-like [31]), } \\
& 1.4 \times 1.4 \times 0.6 \mathrm{~km}^{3} \quad \text { (NEMO-like [32]) }
\end{aligned}
$$

In Table 1 the corresponding double-bang rates for lower, upper and both hemispheres are shown.

TABLE $1 . \quad$ Number of totally contained doublebang events in $\mathrm{km}^{3}$ detector per year.

\begin{tabular}{cccc}
\hline \hline Spectrum & Rate $\left(N_{-2 \pi} / N_{2 \pi} / N_{4 \pi}\right)$ \\
\hline$[21]$ & 1.0 & 2.1 & 3.1 \\
{$[18]$} & 1.4 & 3.1 & 4.5 \\
\hline$[21]$ & \multicolumn{3}{c}{ IceCube-like } \\
{$[18]$} & 0.7 & 1.6 & 2.3 \\
\hline
\end{tabular}

Calculated values for upper hemisphere are $3-6$ times lower compared to [26] since more optimistic predictions for diffuse neutrino fluxes were used there.

Finally, we found using the theoretical predictions in $[18,21]$ for AGN diffuse neutrino fluxes that in a $1 \mathrm{~km}^{3}$ neutrino telescope one can expect a marginally observable rate of double-bang events from $\tau$-neutrinos.

[1] J. G. Learned and S. Pakvasa, Astropart. Phys. 3, 267 (1995).

[2] F. Halzen and D. Saltzberg, hep-ph/9804354 v.1.

[3] J. G. Learned and K. Mannhaim, Ann. Rev. Nucl. Part. Sci., 50, 679 (2000). 
[4] G. Barenboin and C. Quigg, Phys. Rev. D 67, 073024 (2003).

[5] H. Athar et al, hep-ph/0112222 v.2.

[6] J. F. Beacom et al, Phys. Rev. Lett., 90181301 (2003).

[7] P. Bhattacharjee and G. Sigl, Phys. Rep. 327, 109 (2000).

[8] I. M. Albuquerque, L. Hiu, and E. W. Kolb, Phys. Rev. D 64, 083504 (2001).

[9] J. H. MacGibbon and B. J. Carr, Astrophys. J. 371, 447 (1991); E. V. Bugaev and K. V. Konishchev, Phys. Rev. D65, 123005 (2002).

[10] J. H. MacGibbon and U. F. Wichoski, hep-ph/0111436 v. 1 .

[11] J. F. Beacom et al, hep-ph/0307025.

[12] R. Engel, D. Seckel, and T. Stanev, Phys. Rev. D 64 093010 (2001); O. E. Kalashev et al, Phys. Rev D 65 103003 (2002).

[13] G. Sigl et al, Phys. Rev. D 59 043504, (1999).

[14] S. Yoshida, G. Sigl, and S. Lee, Phys. Rev. Lett. 815505 (1998); T. J. Weiler, Astropart. Phys. 11, 303 (1999).

[15] J. L. Feng et al, Phys. Rev. Lett. 88161102 (2002).

[16] T. Abu-Zayyad et al, Astrophys. J. 557, 686 (2001).

[17] Pierre Auger Observatory http://www.auger.org.

[18] K. Mannheim, R. J. Protheroe, and J. P. Rachen, Phys. Rev. D 63, 023003 (2001).

[19] K. Mannheim, Astropart. Phys. 3, 295, (1995).

[20] F. Halzen and E. Zas, Astrophys. J. 488, 669 (1997).

[21] R. J. Protheroe, in Accretion Phenomena and Related Outflows, v.163 of IAU Colloquium, ed. by D. Wickramasinghe et al, (The Astron. Soc. of the Pacific, 1997) p. 585.

[22] F. W. Stecker and M. H. Salamon, Space Sci. Rev. 75, 341, (1996).

[23] A. A. Zdziarski, Mon. Not. Roy. Astr. Soc. 281, L9 (1996).

[24] J. Ahrenc et al, astro-ph/0303218.

[25] V. Aynutdinov et al astro-ph/0305302.

[26] H. Athar et al, Phys. Rev. D62 093010 (2000).

[27] S. I. Dutta et al Phys. Rev. D 62123001 (2000); S. I. Dutta et al Phys. Rev. D 66077302 (2002).

[28] I. A. Sokalski, E. V. Bugaev, and S. I. Klimushin, Phys. Rev. D 64074015 (2001).

[29] E. V. Bugaev and Yu. Shlepin, Phys. Rev. D 67034027 (2003).

[30] E. V. Bugaev, T. Montaruli, and I. A. Sokalski, astroph/0305284.

[31] IceCube project, http://icecube.wisc.edu.

[32] NEMO project, http://nemoweb.lns.infn.it. 